\title{
A NOTE ON NEGATIVE ISOTROPIC CURVATURE
}

\author{
HARISH SESHADRI
}

\begin{abstract}
We prove that any smooth orientable closed four-manifold admits a Riemannian metric with negative isotropic curvature in the sense of Micallef and Moore.
\end{abstract}

\section{Introduction}

An interesting notion of curvature, that of curvature on isotropic 2-planes, was introduced by Micallef and Moore in [5]. The definition is as follows. Let $(M, g)$ be a Riemannian manifold of dimension at least 4 . Consider the complexification $T M \otimes_{\mathbb{R}} \mathbb{C}$. The inner products on $T M$ and $\wedge^{2} T M$ extend to complex bilinear maps on $T M \otimes \mathbb{C}$ and $\wedge^{2} T M \otimes \mathbb{C}$ and we denote these extensions by $\langle$,$\rangle in$ both cases. A subspace $W \subset T_{p} M \otimes \mathbb{C}$ is said to be isotropic if $\langle w, w\rangle=0$ for all $w \in W$. Now let $R: \wedge^{2} T M \otimes \mathbb{C} \rightarrow \wedge^{2} T M \otimes \mathbb{C}$ denote the complex linear extension of the curvature operator of $g$. The metric $g$ is said to have positive (resp. negative) isotropic curvature if $\langle R(v \wedge w), \bar{v} \wedge \bar{w}\rangle>0$ (resp. $<0$ ) whenever $\{v, w\}$ span an isotropic 2-plane.

Considerations of the second variation of area of minimal surfaces lead naturally to isotropic curvature. Examples of manifolds with isotropic curvature of a fixed sign are pointwise quarter-pinched manifolds and manifolds with positive or negative curvature operators.

The relationship between the sign of isotropic curvature and the "classical curvatures" is somewhat mysterious. It is known (cf. [6]) that positive isotropic curvature does not imply positive Ricci curvature but does imply positive scalar curvature (a similar statement is true for negative isotropic curvature). As in the case of positive scalar curvature, one knows that if two manifolds have positive isotropic curvature then their connected sum admits a metric with positive isotropic curvature. However, unlike the case of positive scalar curvature, surgeries in lower codimension need not preserve positive isotropic curvature.

Pursuing the analogy between isotropic and scalar curvatures, one is tempted to ask whether every smooth closed manifold admits a metric with negative isotropic curvature. In this note we show that this is the case in dimension 4.

Theorem 1.1. Any smooth orientable closed four-manifold admits a $C^{\infty}$ metric with negative isotropic curvature.

Received November 25, 2003.

2000 Mathematics Subject Classification. Primary 53C21. 


\section{Remarks:}

(i) A Kähler metric can never have negative isotropic curvature. In fact, if $v$ and $w$ are such that $(v \wedge w)^{1,1}=0$, then $\langle R(v \wedge w), \bar{v} \wedge \bar{w}\rangle=0$. There exist $v$ and $w$ such that $(v \wedge w)^{1,1}=0$ and $\mathbb{C}\{v, w\}$ is an isotropic 2-plane [3].

(ii) In the definition of isotropic curvature, if one demands that $\langle R(v \wedge w), v \wedge$ $w\rangle<0$ for any $v \wedge w \in \wedge^{2} T M \otimes \mathbb{C}$, then one says, following Sampson [8], that $g$ has negative Hermitian sectional curvature. This curvature occurs in the study of harmonic maps of Kähler manifolds and clearly implies negative sectional curvature. Hence it has strong topological implications while Theorem 1.1 states that negative isotropic curvature, in dimension 4 at least, has no topological implications.

The proof of Theorem 1.1 essentially hinges on the fact that in dimension four, the negative isotropic curvature condition can be rephrased as a variational problem. The functional involved will be the integral of a scalar curvature-like function $\sigma$ and its domain will be the space of metrics. Once this is noted the proof involves showing that the functional becomes negative for a suitable choice of metric $g$. Then standard techniques in conformal geometry (as in [2]) can be applied to show that there exists a conformal deformation of $g$ for which $\sigma$ is negative. The geometric part of the proof is the construction of $g$. We do this by gluing in a product of a circle with a hyperbolic 3-manifold of finite (but large) volume. This hyperbolic 3-manifold will be a knot complement in $S^{3}$ and the gluing will be done without changing the smooth topology of $M$.

Note that the proof below only gives a $C^{2, \alpha}$ metric with negative isotropic curvature. The density of $C^{\infty}$ metrics in the space of $C^{2, \alpha}$ metrics (with the $C^{2, \alpha}$ norm) will then give us a $C^{\infty}$ metric with negative isotropic curvature.

\section{Proof}

Proof. For the sake of exposition we divide the proof into two steps.

STEP 1: Let $M$ be a closed smooth four-manifold. A simple calculation, as in [5], shows that a metric $g$ on $M$ has negative isotropic curvature if and only if

$$
\frac{s}{6} I-W<0,
$$

where $s$ denotes the scalar curvature, $I$ is the identity operator and $W$ the tracezero symmetric operator given by Weyl curvature on $\wedge^{2} T M$. To prove (1) it is enough to show that

$$
\sigma_{g}:=\frac{s}{6}+|W|<0,
$$

where $|W|$ denotes the pointwise norm of $W$ (regarded as an element of $\left.\otimes^{4} T^{*} M\right)$. This is because $W$ is a symmetric operator and hence $\langle W(\alpha), \alpha\rangle \geq-|W||\alpha|^{2}$.

The main property of $\sigma_{g}$ is that under conformal changes it transforms like scalar curvature. More precisely, if $g \rightarrow \tilde{g}=u^{\frac{4}{n-2}} g$, the modified scalar curvature 
transforms (in what follows, the Laplacian $\triangle=-d^{*} d$ ) by

$$
\sigma_{g} \rightarrow \sigma_{\tilde{g}}=u^{\frac{-4}{n-2}} \sigma_{g}-4 \frac{n-1}{n-2} u^{-\frac{n+2}{n-2}} \triangle u .
$$

This follows immediately from the corresponding transformation law for the usual scalar curvature $s$ cf. [1] and the fact that $\left|W_{f^{2} g}\right|_{f^{2} g}=f^{-2}\left|W_{g}\right|_{g}$ for any smooth positive function $f$.

Consider the functional on the space of $C^{2}$ metrics on $M$ defined by

$$
F(g)=\int_{M} \sigma_{g} d V_{g}
$$

where $d V_{g}$ is the volume form of the metric $g$. A standard argument, which we outline below, shows that if $F(g)<0$ for some $g$, then there is a $C^{2, \alpha}$ metric $\tilde{g}$ in the conformal class of $g$ with $\tilde{\sigma}_{g}<0$.

Define the operator $L$ on $C^{\infty}(M)$ by

$$
L=-4 \frac{(n-1)}{(n-2)} \triangle+\sigma_{g}
$$

Let $<,>$ denotes the $L^{2}$ inner product on $C^{\infty}(M)$. and let

$$
\lambda=i n f_{\substack{f \in W^{1,2} \\\|f\|_{2}=1}}<L f, f>
$$

and $u$ be the corresponding eigenfunction. We note that since $\sigma_{g}$ is, in general, Lipschitz continuous but not smooth (at the zero locus of $|W|$ ), the best regularity we can obtain for $u$ is that $u \in C^{2, \alpha}$ for any $0<\alpha<1$. This is sufficient for our purposes. By the minimum principle $u>0$ and by definition, $u$ satisfies

$$
L(u)=\lambda u \text {. }
$$

Now suppose that $g$ satisfies $F(g)<0$. Then $<L(1), 1>=F(g)<0$. Hence $\lambda<0$.

Consider the metric $\tilde{g}=u^{\frac{4}{n-2}} \tilde{g}$. By (2) and (3), we see that

$$
\sigma_{g}=u^{-\frac{(n-2)}{4(n-1)}-1} L(u)<0 .
$$

Hence we are reduced to constructing a metric $g$ with $F(g)<0$. This is done in the next step.

STEP 2: Let $K$ be a knot in $S^{3}$ such that $N:=S^{3}-K$ admits a complete hyperbolic metric (constant curvature -1$) g_{H}$ of finite volume. Such a knot exists by the work of Riley [7]. Let $\bar{N}=S^{3}-\nu(K) \subset N$, where $\nu(k)$ is an open tubular neighbourhood of $K$. Fix an embedding of $\bar{N} \times S^{1}$ in $M$ (which exists since $S^{3}-K$ can be embedded in a 3 -ball).

Briefly, we will construct metrics, depending on a parameter $c$, on $\bar{N} \times S^{1}$ which are isometric to a fixed standard metric near $\partial\left(\bar{N} \times S^{1}\right)$. Then these metrics can be extended to all of $M$ such that the extensions agree on $M-(\bar{N} \times$ $\left.S^{1}\right)$. For large values of $c$ we get metrics for which $F<0$. 
The actual construction is as follows: $N$ has one cusp end and this end is isometric to $\left(E:=[0, \infty) \times T^{2}, g_{H}:=d t^{2}+e^{-2 t} g_{e}\right)$, where $T^{2}$ is the 2-torus and $g_{e}$ a flat metric on it. Now consider the metrics $c^{2} g_{H}$, where $c \in \mathbb{R}^{+}$on $N$. We note two features of these metrics.

(i) The sectional curvatures of these metrics go to zero as $c \rightarrow \infty$. Also, by choosing $a(c)$ appropriately (in fact $a(c)=c \log \left(\frac{c}{2}\right)$ will work, see (6) below), we can ensure that the "cross-sectional" torii are approximately of fixed size on the portion $[a(c), a(c)+1] \times T^{2}$ of the end.

(ii) For any $\alpha \geq 0$, let $N_{\alpha}:=N-\left([\alpha, \infty) \times T^{2}\right)$. Then

$$
\int_{N_{0}} s_{c^{2} g_{H}} d V_{c^{2} g_{H}}=-6 c V \operatorname{ol}\left(N_{0}, g_{H}\right) \rightarrow-\infty \text { as } c \rightarrow \infty .
$$

Now the scaled metric $c^{2} g_{H}$ on the end can be written as

$$
c^{2} g_{H}:=d t^{2}+c^{2} e^{-2 \frac{t}{c}} g_{e} \text { with } t \in[0, \infty) .
$$

We deform $c^{2} g_{H}$ to the product (flat) metric $d t^{2}+g_{e}$ in the interval $[a(c), a(c)+1]$, $a(c)$ to be specified, as follows: let $\phi: \mathbb{R} \rightarrow[0,1]$ be a smooth decreasing function such that

$$
\begin{aligned}
\phi(t) & =1 \text { for } t \leq 0 \\
& =0 \text { for } t \geq \frac{1}{2} .
\end{aligned}
$$

Further, let

$$
f_{c}(t)=\phi(t-a(c)) c e^{-\frac{t}{c}}+1-\phi(t-a(c)) .
$$

Then the metric

$$
k_{c}=d t^{2}+f_{c}(t)^{2} g_{e}, \quad t \in[0, \infty)
$$

deforms $c^{2} g_{H}$ to $d t^{2}+g_{e}$ over the interval $[a(c), a(c)+1]$.

For $c>\frac{1}{\log (2)}$, let $a(c)=c \log (c)$. If $t \in[a(c), a(c)+1]$, then $\frac{1}{2}<c e^{-\frac{t}{c}}<1$. From this an easy computation shows that the $C^{2}$ distance between the metrics $k_{c}$ (defined in (5)) and $d t^{2}+g_{e}$ on $[a(c), a(c)+1] \times T^{2}$ is bounded,

$$
\left|k_{c}-\left(d t^{2}+g_{e}\right)\right|_{C^{2}} \leq A,
$$

with $A$ independent of $c$.

The metric $g_{c}$ that we are interested (on $M$ ) is now given by:

$$
\begin{aligned}
g_{c} & =c^{2} g_{H}+d \theta^{2} \text { on } N_{0} \times S^{1} \\
& =k_{c}+d \theta^{2} \text { on }[0, a(c)+1] \times T^{2} \times S^{1} \\
& =\bar{g} \text { on } M-\left(N_{a(c)+1} \times S^{1}\right),
\end{aligned}
$$

where $\bar{g}$ is a fixed extension of the standard product metric $d t^{2}+g_{e}+d \theta^{2}$ on a tubular neighbourhood of $T^{2} \times S^{1} \cong \partial\left(M-\left(\bar{N} \times S^{1}\right)\right) \cong \partial\left(M-\left(N_{a(c)+1} \times S^{1}\right)\right)$, 
where $\cong$ denotes diffeomorphism, to all of $M-\left(\bar{N} \times S^{1}\right)$. This extension can obviously be chosen to be independent of $c$.

Now we claim that $F\left(g_{c}\right)<0$ for large $c$. To see this, note first that the scalar curvature integral $\int_{M} s_{g_{c}} d V_{g_{c}}$ is equal to

$$
\int_{N_{a(c)} \times S^{1}} s_{g_{c}} d V_{g_{c}}+\int_{[a(c), a(c)+1] \times T^{2} \times S^{1}} s_{g_{c}} d V_{g_{c}}+\int_{M-\left(\bar{N} \times S^{1}\right)} s_{g_{c}} d V_{g_{c}},
$$

where we have identified $N_{a(c)+1}$ with $\bar{N}$ in the last integral. Now, as $c \rightarrow \infty$, the first integral goes to $-\infty$ by (4). The second remains bounded by (6) and the third is independent of $c$. Hence

$$
\int_{M} s_{g_{c}} d V_{g_{c}} \rightarrow-\infty \text { as } c \rightarrow \infty
$$

Similarly, the Weyl curvature integral $\int_{M}\left|W_{g_{c}}\right| d V_{g_{c}}$ is equal to

$$
\int_{N_{a(c)} \times S^{1}}\left|W_{g_{c}}\right| d V_{g_{c}}+\int_{[a(c), a(c)+1] \times T^{2} \times S^{1}}\left|W_{g_{c}}\right| d V_{g_{c}}+\int_{M-\left(\bar{N} \times S^{1}\right)}\left|W_{g_{c}}\right| d V_{g_{c}},
$$

where the norm of $W_{g_{c}}$ is with respect to $g_{c}$. Again, for the same reasons, the second and third integrals will remain bounded as $c \rightarrow \infty$. To see that the first integral is zero, recall that the metric on $N_{a(c)} \times S^{1}$ is the product metric and the product of a constant sectional curvature metric with $S^{1}$ is conformally flat. Hence

$$
\int_{M}\left|W_{g_{c}}\right| d V_{g_{c}} \text { remains bounded as } c \rightarrow \infty \text {. }
$$

Combining (8) and (10) we see that $F\left(g_{c}\right)<0$ for large $c$.

\section{Remarks:}

(i) The proof above actually shows that on any closed 4-manifold, for any $\mu>0$, there exists a metric $g$ with $\mu s_{g}+\left|W_{g}\right|<0$.

(ii) In the case of negative scalar curvature, one knows by the work of Lohkamp [4], that the space of such metrics $\mathfrak{M}$ is contractible. One can ask if the same is true for the space of negative isotropic curvature metrics. Note that in Lohkamp's paper, a variational approach similar to Step 1 is used to extend a map of $S^{n}$ into $\mathfrak{M}$ to a map of $B^{n+1}$ (in order to prove that $\pi_{n}(\mathfrak{M})=0$ ).

(iii) The proof hinges on the fact that in dimension 4, there exists an expression of the form $\mu s_{g} \mp\left|W_{g}\right|$, with $\mu>0$ such that if this expression has a certain fixed sign, then the isotropic curvature also has the same sign. In order to extend the proof to dimensions $n \geq 5$, one needs to know if a similar statement is true for all $n$. However the following example, given in [6] shows that such a statement is not true in the positive case. The product metric on $M=\Sigma \times S^{2 m}$, where $\Sigma$ is a compact surface of constant curvature -1 and $S^{2 m}$ is the unit sphere of dimension $2 m \geq 4$, is conformally flat and has positive 
scalar curvature. However, since $H_{2}(M, \mathbb{R}) \neq 0$ by Theorem 2.1 of $[6], M$ does not admit a metric of positive isotropic curvature.

\section{Acknowledgements}

I would like to thank the referee for pointing out various errors in the original draft.

\section{References}

[1] A. L. Besse, Einstein Manifolds, Springer-Verlag, Berlin-Heidelberg, 1978.

[2] M. J. Gursky, Four-manifolds with $\delta W^{+}=0$ and Einstein constants of the sphere, Math. Ann. 318 (2000), 417-431.

[3] A. El Soufi, R. Petit, Immersions minimales et immersions pluriharmoniques entre variétés Riemanniennes: résultats de non existence et de rigiditeé, Ann. Inst. Fourier (Grenoble), 50 (2000), 235-256.

[4] J. Lohkamp, The space of negative scalar curvature metrics, Invent. Math. 110 (1992), 403-407.

[5] M. J. Micallef and J. D. Moore, Minimal two-spheres and the topology of manifolds with positive curvature on totally isotropic two-planes, Ann. of Math. 127 (1988), 199-227.

[6] M. J. Micallef and M. Y. Wang, Metrics with nonnegative isotropic curvature, Duke Math. Journal, 72 (1993), 649-672.

[7] R. Riley, A quadratic parabolic group, Math. Proc. Cambridge Philos. Soc., 77 (1975), $281-288$.

[8] J. H. Sampson, Application of harmonic maps to Kähler geometry, Contemp. Math., 77 (1984), 125-134.

E-mail address: harish@isibang.ac.in

Stat-Math Unit,, Indian Statistical Institute, Bangalore, India 